Review Article

\title{
Effect of Acupuncture on Neuroplasticity of Stroke Patients with Motor Dysfunction: A Meta-Analysis of fMRI Studies
}

\author{
Qiuyi Lv $\mathbb{D}^{1},{ }^{1}$ Guixing Xu $\mathbb{D}^{2}{ }^{2}$ Yuxin Pan $\mathbb{D}^{3}{ }^{3}$ Tongtong Liu $\mathbb{D}^{4},{ }^{4}$ Xiaodong Liu $\left(\mathbb{D},{ }^{5}\right.$ \\ Linqing Miao $\mathbb{D}^{6}{ }^{6}$ Xing Chen $\mathbb{D}^{1},{ }^{1}$ Lan Jiang, ${ }^{1}$ Jie Chen, ${ }^{7}$ Yingjia He $\mathbb{D},{ }^{5}$ Rong Zhang $\mathbb{D}^{5}$ \\ and Yihuai Zou ${ }^{1}$ \\ ${ }^{1}$ Department of Neurology and Stroke Center, Dongzhimen Hospital, The First Affiliated Hospital of Beijing University of \\ Chinese Medicine, Beijing, China \\ ${ }^{2}$ The Acupuncture and Tuina School/The 3rd Teaching Hospital, Chengdu University of Traditional Chinese Medicine, \\ Chengdu, China \\ ${ }^{3}$ Institute of Neuroscience, Chinese Academy of Sciences, Shanghai, China \\ ${ }^{4}$ Guang'anmen Hospital, China Academy of Chinese Medical Sciences, Beijing, China \\ ${ }^{5}$ Beijing University of Chinese Medicine, Beijing, China \\ ${ }^{6}$ Beijing Institute of Technology, Beijing, China \\ ${ }^{7}$ School of Life Science, Peking University, Beijing, China
}

Correspondence should be addressed to Yihuai Zou; zouyihuai2004@163.com

Received 26 June 2020; Revised 17 March 2021; Accepted 21 May 2021; Published 3 June 2021

Academic Editor: Yasuo Terao

Copyright ( 2021 Qiuyi Lv et al. This is an open access article distributed under the Creative Commons Attribution License, which permits unrestricted use, distribution, and reproduction in any medium, provided the original work is properly cited.

\begin{abstract}
Importance. Acupuncture is an effective treatment for stroke, especially in the aspect of motor deficit. Many brain imaging studies of acupuncture have found significant changes in brain function after acupuncture treatment in order to reveal its underlying mechanisms in regulating neural plasticity. However, no definite consensus has been reached. Objective. To analyze the pattern of intrinsic brain activity variability that is altered by acupuncture compared with conventional treatment in stroke patients with motor dysfunction, thus providing the mechanism of stroke treatment by acupuncture. Methods. Chinese and English articles published up to May 2020 were searched in the PubMed, Web of Science, EMBASE, and Cochrane Library databases, China National Knowledge Infrastructure, Chongqing VIP, and Wanfang Database. We only included randomized controlled trials (RCTs) using resting-state fMRI to observe the effect of acupuncture on stroke patients with motor dysfunction. R software was used to analyze the continuous variables, and Seed-based d Mapping with Permutation of Subject Images (SDM-PSI) was used to perform an analysis of fMRI data. Findings. A total of 7 studies comprising 143 patients in the treatment group and 138 in the control group were included in the meta-analysis. The results suggest that acupuncture treatment helps the healing process of motor dysfunction in stroke patients and exhibits hyperactivation in the bilateral basal ganglia and insula and hypoactivation in motor-related areas (especially bilateral BA6 and left BA4). Conclusion. Acupuncture plays a role in promoting neuroplasticity in subcortical regions that are commonly affected by stroke and cortical motor areas that may compensate for motor deficits, which may provide a possible mechanism underlying the therapeutic effect of acupuncture.
\end{abstract}

\section{Introduction}

Stroke is a syndrome characterized by an acute onset of neurologic deficit resulted from ischemia or hemorrhage. It became the leading cause of death and years of life lost (YLLs) at the national level in China [1]. Patients with stroke are susceptible to several complications, including pain, dysphagia, pneumonias, incontinence, depression, and somatic dyskinesia, which usually impede clinical recovery and require specific intervention $[2,3]$. In this review, we mainly focus on the motor dysfunction after stroke. As a common and severe complication of stroke, motor dysfunction recovery requires 
multidisciplinary treatment and still remains a clinical challenge [4]. Seeking an effective and safe alternative therapy meets the requirement of the Healthy China 2030 plan [5].

Acupuncture, a key modality of Traditional Chinese Medicine (TCM) therapy, is recommended by the World Health Organization (WHO) as an alternative and complementary strategy for poststroke treatment $[6,7]$. Acupuncture has been widely used to relieve pain and improve motor or neurological function $[8,9]$. Sufficient previous studies including animal experiments, clinical trials, and systematic reviews have proven that acupuncture was beneficial for stroke rehabilitation [10]. Although the application of acupuncture has shown good clinical efficacy, the mechanisms of acupuncture remain unknown. One hypothesis suggests that acupuncture may induce neuroplasticity to exert therapeutic effects [11-13].

Neuroplasticity refers to the capability of the nervous system to modify and reorganize the brain structure and function in response to impairment or experience; various behavioral, neurophysiological, and neuroimaging studies can document changes in the nervous system $[14,15]$. One of the recommended modalities used to measure neuroplasticity in humans is resting-state functional magnetic resonance imaging (rs-fMRI), which is often used to assess resting-state networks in participants $[16,17]$. The main analysis approaches of rs-fMRI are regional homogeneity (ReHo) and amplitude of low-frequency fluctuations (ALFFs). The former evaluates the temporal homogeneity of the regional blood oxygen level-dependent (BOLD) signal, and the latter reflects intrinsic brain low frequency $[18,19]$. Aimed at a better understanding of the cerebral characters of acupuncture treatment, more and more acupuncture studies have applied rs-fMRI in the clinical trials. However, no consensus has been reached, possibly due to the small sample size or distinctions of experimental design between studies. For example, Schaechter et al. [20] have reported that acupuncture would activate the ipsilateral lesional motor cortex to improve motor function in patients with hemiparetic stroke, while others suggested that the basal ganglia would be predominantly activated by acupuncture [21].

Therefore, in order to elucidate which brain area plays a crucial role in the therapeutic effect of acupuncture, this review synthesized corresponding studies and introduced coordinate-based meta-analysis (CBMA) to quantitatively integrate the results of individual neuroimaging studies $[22,23]$. The feature of CBMA is the use of effect sizes, which allows combination of peak coordinates with statistical parametric maps and it has been fully validated in many studies [24-28]. In the present study, we applied the Seedbased $d$ Mapping with Permutation of Subject Images (SDM-PSI), which is a new generation algorithm for CBMA meta-analysis, to analyze the rs-fMRI data of included studies $[29,30]$. The results would determine whether acupuncture has a differential effect on brain activity as compared to regular or conventional treatment. The apprehension of neuroplasticity mechanisms induced by acupuncture can not only elucidate the central mechanism of acupuncture but also possibly provide novel ideas for applying acupuncture to the treatment of other neurodegenerative diseases.

\section{Methods}

We conducted the meta-analysis in line with the Cochrane Handbook for Systematic Reviews of Interventions. All procedure followed the Preferred Reporting Items for Systematic Reviews and Meta-Analyses guidelines (PRISMA guidelines). The study was registered in the PROSPERO International prospective register of systematic reviews (registration number: CRD 42020185421).

2.1. Literature Search. Studies that examined the neuroprotective effect of acupuncture in stroke patients were included in the present study. The PubMed, EMBASE, and Cochrane Library databases, Web of Science, China National Knowledge Infrastructure (CNKI), Chongqing VIP (VIP), and the Wanfang Database (WF) were searched from inception until May 2020 by two independent researchers. The following English search terms were used: (stroke OR Poststroke OR Cerebrovascular Accident OR Cerebrovascular Apoplexy OR Apoplexy OR Brain Vascular Accident OR Cerebrovasc* OR brain* OR brain vasc* OR hemipleg* OR apoplex* OR CVA OR TIA) AND (acupuncture OR acupuncture therapy OR acupuncture treatment OR electroacupuncture OR electro-acupuncture OR acupuncture, ear OR ear acupuncture OR auriculotherapy OR scalp acupuncture) AND (RCT OR randomized controlled trial OR controlled clinical trial OR randomized OR clinical trial OR randomly OR RCT OR trial) AND (fMRI OR functional MRI OR functional magnetic resonance imaging $\mathrm{OR}$ neuroimaging). The languages of the trials were restricted to English or Chinese, and the search strategy for each database was based on its own unique characteristics.

2.2. Inclusion/Exclusion Criteria. Two researchers independently conducted the search and screened the titles, abstracts, and full texts of the papers. Studies were included based on the following criteria: (1) an RCT conducted in patients with stroke sequelae at any-stroke stage, of any age and gender; (2) manual acupuncture or electroacupuncture with or without other therapies in the treatment group, while other therapies including conventional rehabilitation or sham acupuncture in the control group; (3) involved whole-brain functional imaging (ReHo or ALFFs) in resting state; (4) threedimensional coordinates $(x, y, z)$ reported in standard stereotactic space Talairach or Montreal Neurological Institute (MNI); (5) if one study involved two or more comparable datasets, all samples were included; and (6) used secondary outcomes to assess clinical efficacy. The exclusion criteria were as follows: (1) the study only used region of interest (ROI) method; (2) the sample size in each group was less than 5 ; (3) if separate papers used the same or similar datasets, only the largest sample was included.

2.3. Data Extraction and Quality Assessment. Data were extracted from the included studies into a standard form with respect to publishing year, name of the first author, sample size, participants' condition, intervention design, fMRI method, and secondary outcome measures. Missing or unclear data for the meta-analysis were obtained after corresponding with the original authors by email or telephone. 
Two independent researchers carried out the data extraction; any disagreements were resolved by a third reviewer. This information is showed in Table 1. Quality assessment was based on Cochrane Risk of Bias Tool and carried out by two researchers independently. All the reports were evaluated according to the following seven criteria: random sequence generation, allocation concealment, blinding of participants and personnel, blinding of outcome assessment, incomplete outcome data, selective reporting, and other sources of bias. For each criterion, studies were judged to be at low, high, or unclear risk of bias.

2.4. Data Synthesis and Meta-Analysis. ReHo results: the differences of brain activity between the treatment group and the control group were analyzed by SDM-PSI (version 6.21, https://www.sdmproject.com/). A step-by-step tutorial is provided on this website. First, reported peak coordinates and effect sizes ( $t$ value, or equivalently from $z$-scores or $P$ values) were extracted to prepare the peaks' text files. In the step of preprocessing, software will use those files to recreate the lower and upper bounds of the possible effect-size values of the studies. Second, the meta-analysis consisted in calculating the random-effects mean of the ReHo values and the mean map was weighted by the sample size and variance of each study. We reported results using family-wise error (FWE) correction $(P<0.05$ and voxel extent $\geq 10)$ with the threshold-free cluster enhancement approach (TFCE) and 5000 permutations. The heterogeneity analyses were also assessed. The peak MNI coordinate would be extracted to derive standard heterogeneity statistics $I^{2}$, and an $I^{2}<50 \%$ indicates low heterogeneity. Funnel plots were not performed because the amount of included studies $(n=7)$ was less than 10, but the Egger test was used to assess the publication bias. Finally, a metaregression analysis was carried out to figure out the relationship between ReHo changes and clinical variables. All results would be reported using the TFCE-based FWE corrected threshold $(P<0.05$ and voxel extent $\geq 10)$.

Clinical variables: statistical analyses of continuous data were performed with R software, version 3.6.2 (R Foundation for Statistical Computing, Vienna, Austria), using the Meta and Metafor meta-analysis packages. Weighted mean difference (WMD) with $95 \%$ CIs was used. Heterogeneity was tested by the $I^{2}$ statistic. We selected fixed or randomeffects model to pool the data depending on whether the trials had good homogeneity $\left(P>0.10, I^{2}<50 \%\right)$ or not. A forest plot was adopted to show the hypothesis test results, and if necessary, a sensitivity analysis or subgroup analysis would be performed to find sources of heterogeneity.

\section{Results}

3.1. Included Studies. The database search yielded 1195 articles. 272 duplicates were removed, and 883 articles were excluded after screening titles and abstracted. Of the 40 potentially relevant reports, 6 articles [31-36] and 7 studies (Chen et al. [35] conducted two trials at the same time) proved eligible after full-text screening (Figure 1).

Trials were published after 2000, and each study enrolled 10 to 100 patients (140 individuals in the treatment group and 138 in the control group). All studied recruited ischemic stroke patients. There were no significant differences in demographic baseline characteristics, including age, gender, disease status including symptom duration, or secondary outcome measures between the two groups. Three studies reported the patient limb dysfunction of the right side and the most lesions of brain regions had the left laterality (especially in the left basal ganglia) $[32,35,36]$. All patients in both groups received conventional treatment and, on this basis, participants in the treatment group underwent two or four courses (weeks) of manual acupuncture therapy, one study combined with sham acupuncture as comparison [32]. The manual acupuncture methods include lifting yang to dredging du meridian needling method (LYDDM) and Xingnao Kaiqiao needling method (XNKQ) and empirical acupoints. The LYDDM and XNKQ needling methods are based on long-term clinical experiments and were developed in by Professor Peilin Zhang and Xuemin Shi, respectively. And the application of empirical acupoints means that doctors manipulate the acupuncture based on their clinical experience. All studies reported rs-fMRI data by using ReHo variations to compare differences before and after treatment in patients from both groups. Clinical efficacy was examined by Fugl-Meyer assessment (FMA; $n=7$ ), nervous functional deficiency scale (NDS; $n=3$ ), Barthel index (BI; $n=4)$, or modified Barthel index (MBI; $n=3$ ). The details and features of the studies are shown in Table 1 .

3.2. Quality Assessment. The quality assessment was based on Cochrane Risk of Bias Tool. Among 7 RCTs, all the studies reported the methods of random sequence generation but only three studies of two articles employed allocation concealment $[31,36]$. Only two studies reported the methods of patient blinding [32, 35], while Zhou et al. [34] admitted that it was at high risk. Methods of blinding of outcome assessment were only applied in two studies [34, 35]. Evaluation of incomplete outcome data depended on whether the clear descriptions of baseline data were shown. According to this standard, all studies were at low risk. None of the studies have published study protocols before, so it was difficult to evaluate selective reporting. We tended to believe a study was at low risk only if it reported all the three secondary outcome measures (FMA, NDS, BI, or MBI). Thus, low risk of bias in the domain of selective reporting was given to four studies [31, 33-35]. In addition, we did not find any other sources of bias. Overall, these studies were not of high quality, mainly in terms of allocation concealment, patient blinding, and outcome blinding. Figure 2 presents the quality assessment of these 7 included studies.

\subsection{Meta-Analysis of ReHo Changes}

3.3.1. Mean Analysis. Patients in the treatment group showed hyperactivation in the right basal ganglia $(P<0.05, z=6.622$ ) and left insula $(P<0.05, z=5.232)$ after acupuncture therapy and the decreased activity of the left precentral gyrus (PG; $P<0.05, z=-4.477$ ) and right superior frontal gyrus (SFG; $P<0.05, z=-4.723$ ). Patients in the control group showed hyperactivation in the right middle frontal gyrus 


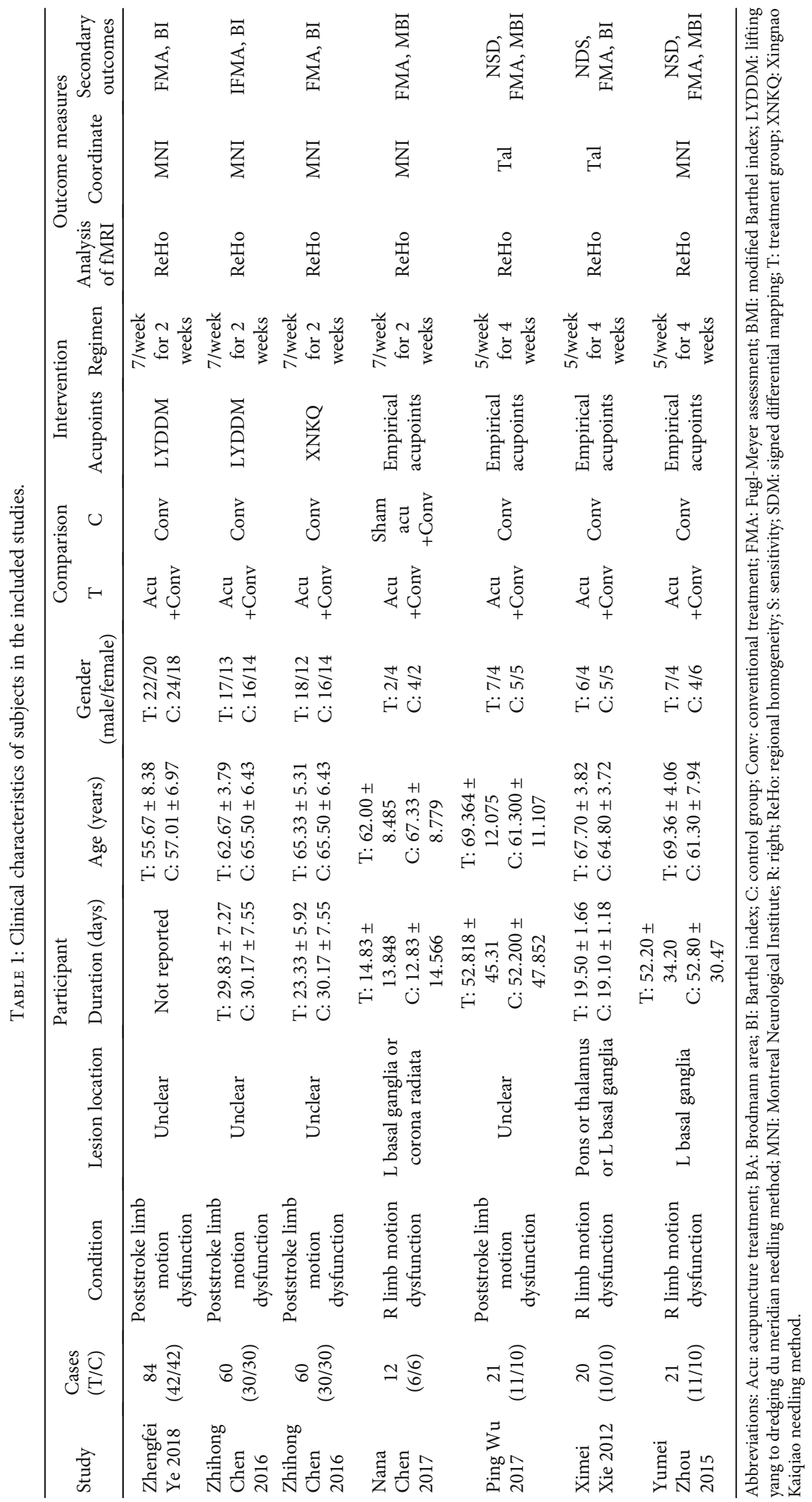




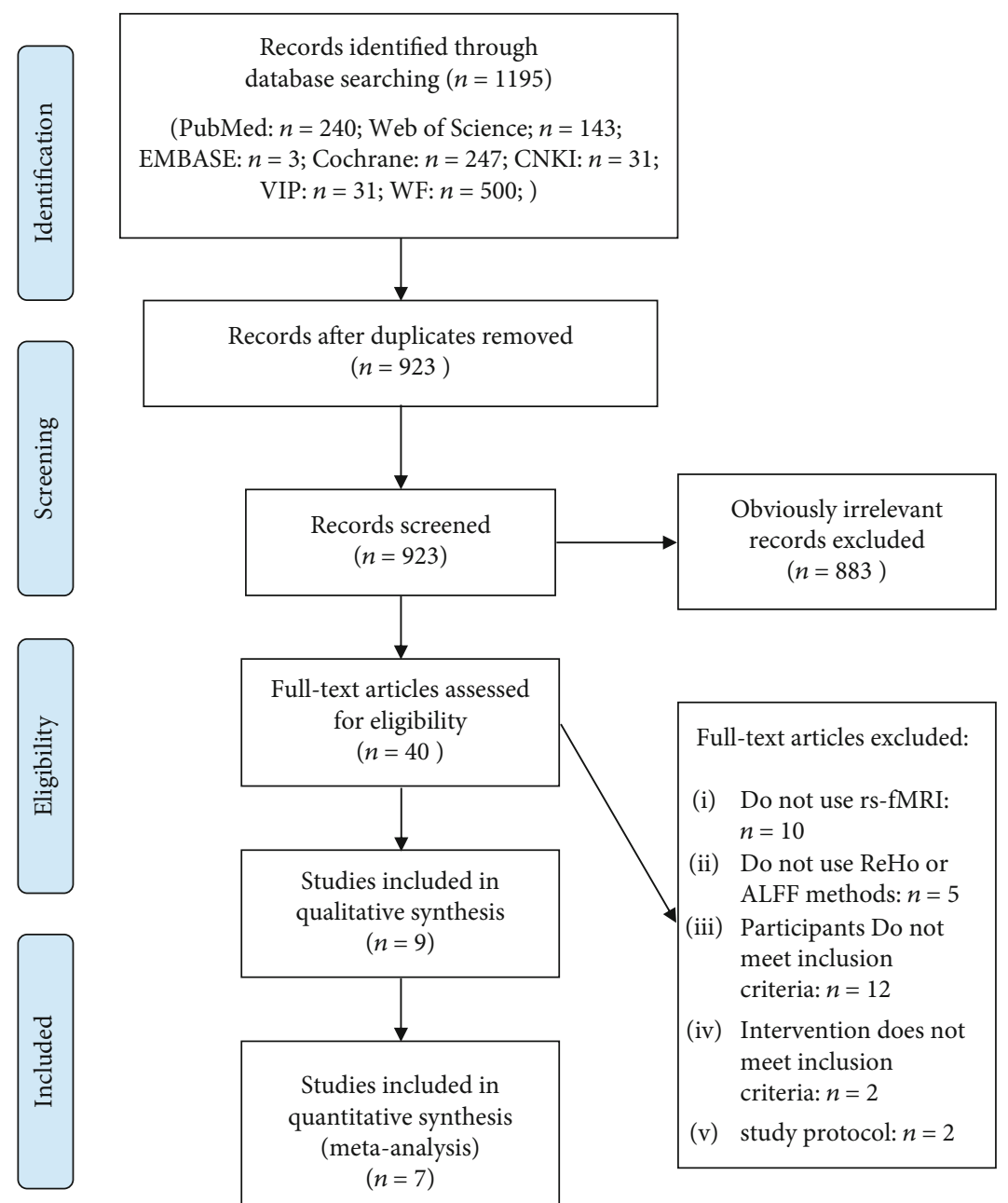

Figure 1: Flowchart for study inclusion and exclusion process.

(MFG; $P<0.05, z=5.457)$ and left supplementary motor area (SMA; $P<0.05, z=4.867$ ) after conventional treatment and a decrease of activity in the left striatum $(P<0.05$, $z=-4.876$ ) and left inferior frontal gyrus (IFG; $P \leq 0.05$, $z=-5.547)$. Peak coordinates and cluster breakdowns are shown in Table 2. Differences in the brain regions between conventional treatment and acupuncture treatment have been visualized in Figure 3.

3.3.2. Heterogeneity Analysis and Publication Bias. In the treatment group, the ganglia, insula, left PG, and right SFG showed low between-study heterogeneity of effect size differences in peak coordinates $\left(I^{2}=0.88 \%-7.40 \%\right)$ and low between-study heterogeneity for each significant peak in the control group $\left(I^{2}=0.09 \%-1.29 \%\right)$. The heterogeneity results are shown in Table 2. The Egger tests were insignificant in the treatment group $(P=0.464)$ and the control group $(P=0.501)$.

3.3.3. Metaregression Analysis. Metaregression was used to search for potential correlation between FMA scores, mean age, and disease duration in the treatment group. The FMA scores are associated with the left insula (Table S1A). The analysis of mean age shows significance in the brain regions of the left cerebellum (Table S1B). The disease duration is associated with many regions, including the left cerebellum, arcuate network and right SMG, and SFG (Table S1C).

3.4. Meta-Analysis of Secondary Outcomes. Compared with conventional treatment, manual acupuncture therapy was associated with a decrease of NDS score $(-4.40 ; 95 \% \mathrm{CI}$, 3.79 to $\left.5.01 ; I^{2}=0\right)$, indicating that acupuncture could mitigate the impairment of nervous function. The change in $\mathrm{BI}$ score was $3.47\left(3.47 ; 95 \% \mathrm{CI}, 0.99\right.$ to $\left.5.95 ; I^{2}=87 \%\right)$ and that in MBI score was $7.49\left(7.49 ; 95 \% \mathrm{CI}, 5.38\right.$ to $\left.9.59 ; I^{2}=0\right)$. The increase of BI or MBI suggested that patients had better daily living ability after acupuncture. A greater increase of FMA score $\left(5.73 ; 95 \% \mathrm{CI}, 4.35\right.$ to $\left.7.11 ; I^{2}=77 \%\right)$ also demonstrated that acupuncture could improve motor function of the upper and lower limb. The forest plots are shown in Figures S1A-S1D.

\section{Discussion}

4.1. Main Findings. It is suggested that acupuncture therapy could relieve neurological deficiency (NDS) and improve 
Random sequence generation

Allocation concealment

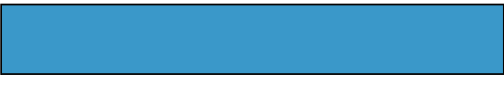

Allocation concealme

Blinding of participants and personnel

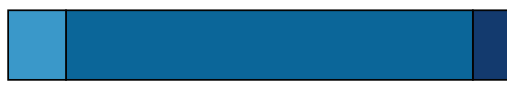

outcome assessment

Incomplete outcome data
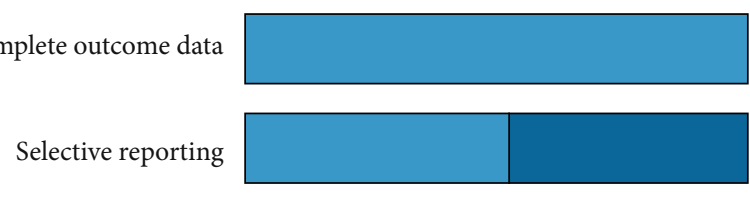

Other source of bias
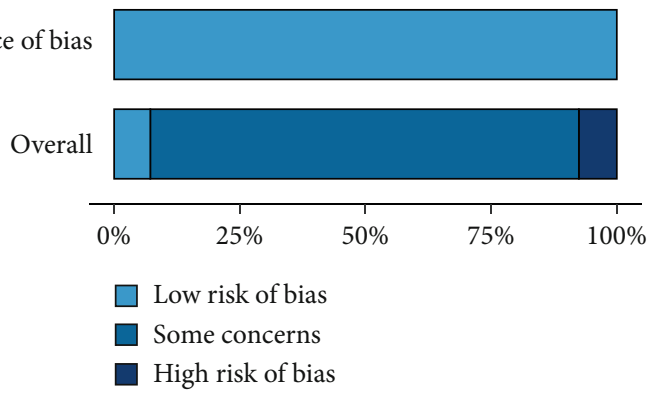

Risk of bias

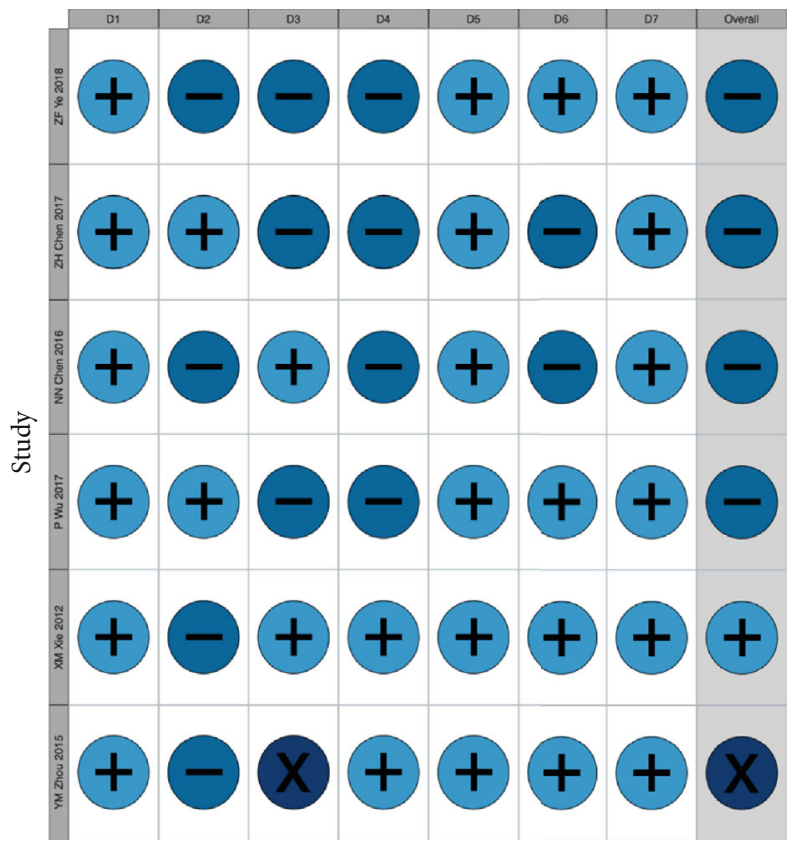

FIGURE 2: Risk of bias summary. Each color represents a level of risk of bias: light blue, low risk of bias; medium blue, unclear risk of bias; dark blue, high risk of bias. D1: random sequence generation; D2: allocation concealment; D3: blinding of participants and personnel; D4: blinding of outcome data; D5: incomplete outcome data; D6: selective reporting; D7: other sources of bias.

TABLE 2: Brain activity changes in patients after treatment compared to baseline.

\begin{tabular}{|c|c|c|c|c|c|c|c|c|}
\hline & \multicolumn{3}{|c|}{$\begin{array}{l}\text { MNI } \\
\text { coordinates }\end{array}$} & \multirow[t]{2}{*}{$\begin{array}{l}\operatorname{SDM} z \\
\text {-score }^{\mathrm{a}}\end{array}$} & \multirow[t]{2}{*}{$\begin{array}{c}P \\
\text { value }^{\mathrm{b}}\end{array}$} & \multirow[t]{2}{*}{ Voxels $^{c}$} & \multirow[t]{2}{*}{ Cluster breakdown } & \multirow[t]{2}{*}{$I^{2}$} \\
\hline & $x$ & $y$ & $z$ & & & & & \\
\hline \multicolumn{9}{|l|}{ Treatment group } \\
\hline $\mathrm{R}$ basal ganglia & 34 & -12 & 8 & 6.622 & $<0.05$ & 1244 & $\begin{array}{c}\mathrm{R} \text { lenticular nucleus, putamen, striatum, insula, rolandic } \\
\text { operculum, BA48 }\end{array}$ & $0.88 \%$ \\
\hline L insula & -32 & -14 & 10 & 5.232 & $<0.05$ & 887 & $\begin{array}{l}\text { L insula, lenticular nucleus, putamen, rolandic operculum, } \\
\text { BA48 }\end{array}$ & $1.02 \%$ \\
\hline L precentral gyrus & -28 & -24 & 66 & -4.477 & $<0.05$ & 337 & $\begin{array}{c}\text { L precentral gyrus, corpus callosum, superior frontal gyrus, } \\
\text { dorsolateral, BA6 }\end{array}$ & $5.83 \%$ \\
\hline $\begin{array}{l}\text { R superior frontal } \\
\text { gyrus }\end{array}$ & 22 & 2 & 62 & -4.723 & $<0.05$ & 20 & Right superior frontal gyrus, dorsolateral, BA6 & $7.40 \%$ \\
\hline \multicolumn{9}{|l|}{ Control group } \\
\hline R middle frontal gyrus & 38 & -6 & 56 & 5.457 & $<0.05$ & 575 & $\begin{array}{l}\text { R middle frontal gyrus, superior frontal gyrus, dorsolateral, } \\
\text { BA6 }\end{array}$ & $0.42 \%$ \\
\hline $\begin{array}{l}\text { L supplementary } \\
\text { motor area }\end{array}$ & -6 & 4 & 66 & 4.867 & $<0.05$ & 83 & L supplementary motor area, BA6 & $1.29 \%$ \\
\hline L basal ganglia & -24 & 0 & -6 & -4.876 & $<0.05$ & 249 & L lenticular nucleus, putamen, striatum, insula, BA48 & $0.09 \%$ \\
\hline $\begin{array}{l}\text { L inferior frontal } \\
\text { gyrus }\end{array}$ & -32 & 32 & -14 & -5.547 & $<0.05$ & 166 & L inferior frontal gyrus, orbital part, BA47 & $0.52 \%$ \\
\hline
\end{tabular}

${ }^{\mathrm{a}}$ Peak height threshold: $z>1 .{ }^{\mathrm{b}}$ Voxel probability threshold: $P<0.005$ uncorrected and remained after correcting threshold (TFCE) of $P<0.05 .{ }^{\mathrm{c}} \mathrm{Cluster}$ extent threshold: number $\geq 10$ voxels. Abbreviations: BA: Brodmann area; $I^{2}$ : heterogeneity $I^{2}$; MNI: Montreal Neurological Institute; R: right; ReHo: regional homogeneity; SDM: signed differential mapping. 


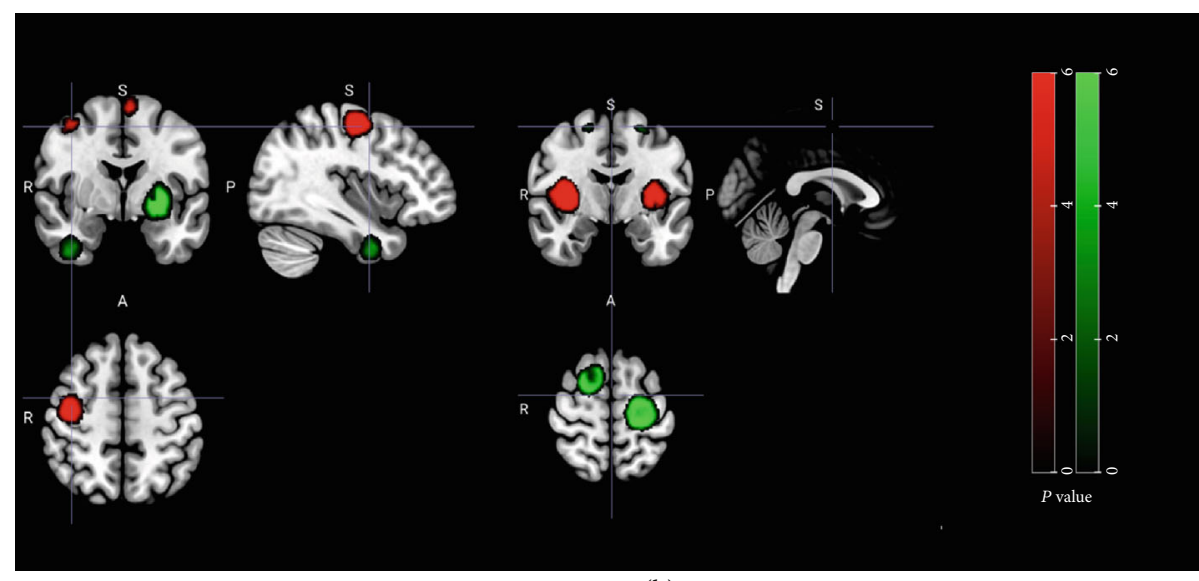

(a)

(b)

FIGURE 3: SDM-PSI meta-analysis for (a) ReHo change in poststroke patients after conventional treatment and (b) ReHo change in poststroke patients after acupuncture treatment. Red color refers to hyperactivation, and green color refers to hypoactivation.

motor function (FMA) and daily living ability (MBI). These findings are discussed in many previous meta-analysis studies, so we will not discuss further here [10, 37, 38]. In this paper, we mainly focus on the meta-analysis of ReHo changes. In the treatment group, stroke patients showed hyperactivation in the bilateral basal ganglia and insula. In the control group, patients showed that brain activities were increased in the bilateral frontal gyrus (BA6). However, the decreased activity of the bilateral frontal gyrus (BA6) and left precentral gyrus (BA4) was found in the treatment group. Additionally, the ReHo index of the left insula significantly correlated with FMA scores across all stroke patients after acupuncture therapy.

4.2. The Involvement of the Basal Ganglia and Insula in Neuroplasticity Induced by Acupuncture. The results showed that resting-state spontaneous brain activity was obviously increased in the bilateral basal ganglia and insula after acupuncture treatment. Hyperactivation in the basal ganglia comprised mostly the striatum. The basal ganglia play an important integrative role in motor regulation [39]. It is implicated in human dystonia and motor control [40]. Wang et al. have compared ischemic stroke patients to healthy participants, and they found that stroke patients showed low ReHo values in the basal ganglia, which is consistent with previous conclusions that the basal ganglia are vulnerable to ischemia $[41,42]$. Regarding motor function, the extent of damage to the basal ganglia seems to be a neural correlate for motor deficit. Three studies included in this review reported that the lesion locations were primarily in the basal ganglia $[32,34,35]$, which is in line with the theory that a common ischemic stroke in deep brain regions featured by high disability rate is basal ganglia lacunar stroke [43]. Thus, it can be speculated that limb motor dysfunction of included ischemic stroke patients was mainly related to basal ganglia impairment. The results revealed that acupuncture therapy was also associated with hyperactivation in the insula. The insula integrates different functional systems including sensory motor and cognition and plays a part in motor pro- graming and control $[44,45]$. Existing evidence suggests that the anterior insula is commonly affected by acute middle cerebral artery stroke [46]. It seems that the damage of the insula is responsible for the motor dysfunction in stroke patients and acupuncture can activate it to exert therapeutic effect. The metaregression analysis also found that the ReHo increases of the insula are positively correlated to the increase in FMA scores, suggesting that acupuncture could improve ability of daily life via the activation of the insula. Here, our results show an increased spontaneous activity in the basal ganglia and insula after acupuncture treatment, indicating that acupuncture could remodel neuronal function of these areas.

4.3. The Involvement of Motor-Related Areas in Neuroplasticity Induced by Acupuncture. In both the treatment and control groups, our results showed significant alterations of brain activation in most parts of motorrelated areas, including the bilateral superior frontal gyrus (especially BA6) and left precentral gyrus (especially BA4). BA4 and BA6 are traditional motor areas, consisting of the "classical" motor network. BA4 is the location of the primary motor cortex (M1), responsible for planning and controlling voluntary motor movement on the body's contralateral side. BA6 includes the premotor cortex (PMC) and supplementary motor area (SMA), where neurons have projections to M1 as well as the corticospinal tract (CST) [47]. It bridges prefrontal and primary motor cortices and is engaged in planning of complex and coordinated movements [48, 49]. Evidence from animal and clinical studies suggests that those areas associate with movement preparation and execution and the activation of these areas contributes to reorganization after stroke [50-52]. Therefore, the functional plasticity of BA6 and BA4 might explain the mechanism of motor recovery.

In this meta-analysis, the ReHo value of bilateral BA6 is significantly increased in the control group but decreased in the treatment group. Here, we speculate that the difference between two groups is in connection with the compensatory 
activity. Compensation at the neuronal level is characterized by activation in alternative brain areas not normally observed in nondisabled individuals [53]. Previous animal studies have demonstrated that reorganization of residual motor cortex contributes to the restoration of movement dysfunction after stroke, which indicates that increased fMRI signal within a given brain area can not only reflect neural recovery but also compensation $[54,55]$. Thus, the increased activation of BA6 in the control group should be interpreted as being associated with compensation, which is in accordance with theories that the increased activity of BA6 is a form of compensation for motor deficit after stroke [56]. A longitudinal fMRI study observed, in the early phase of poststroke recovery (less than 6 months), hyperactivation in bilateral SMA and PMC [57]. Another study with similar results identified a positive correlation, involving increased signals in SMA, cingulate, and other motor areas of both hemispheres with good recovery process (from 2 to 7 weeks up to 6 months), suggesting that compensatory network may be the substrates of rehabilitation strategies [58]. All the 7 studies in this review were similar in the duration of symptoms, ranging from 9-14 days up to 6 months, at which status the activation of BA6 would be increased according to the conclusions above. Interestingly, after receiving acupuncture treatment, the activation level of bilateral BA6 is induced to reduce. The decreased activity of BA6 may implicate the reduced dependence on its compensation, indicating the recovery of motor network, thus helping the ipsilateral limb to recover. Similarly, the results revealed that acupuncture therapy was also associated with hypoactivation in left BA4. Evidence from meta-analysis describes that an increased activation in contralesional M1 (BA4) is a highly consistent finding across different impairment levels and times poststroke [59]. This is in accordance with theories that the increased activity of sound side hemisphere is a form of compensation for dysfunction of other damaged cerebral cortex [56]. From this perspective, if most brain lesions of included patients had the right laterality, we would expect to see a weakened compensation of contralesional BA 4 by acupuncture. Considering that 4 studies in this review did not report the locations of infarcted lesions, this assumption warrants further experiments and opens a way for investigation of the relationship between acupuncture treatment and compensatory activity of BA4.

4.4. Strengths and Limitations. This review is unique and innovative. To our knowledge, we are the first to have SDM-PSI analysis of rs-fMRI used in RCTs of acupuncture. Altered cerebral activity in ischemic stroke patients with motor dysfunction provides a new insight into brain mechanism and clinical application, and this review is intended to serve as both a challenge and an encouragement. Also, strict inclusion criteria were made to minimize selection bias, and 7 studies were synthesized in the final analysis. These trials generally consisted of similar target participants and study designs, thus making results comparable.

As an innovative study, this systematic review still has certain limitations. The main limitation of this review is the small sample size. Only 7 trials and 278 participants met the inclusion criteria so that publication bias and subgroup analysis of secondary outcomes measures were not performed. The results of quality assessments indicated that some studies were of low quality, especially in terms of blinding. The lack of high-quality RCTs made it hard to perform an accurate analysis of the interaction between acupuncture efficacy and neuroplasticity. Thus, to better figure out the potential influence of acupuncture on neuroplasticity after stroke, it is time to organize large-scale and elaboratedesigned RCTs for further investigations. Moreover, it is supposed that different acupuncture parameters (referring to different acupoints, duration, frequency, and operation methods) may cause fine distinctions of altered cerebral activity. Therefore, there may exist heterogeneity in the acupuncture therapy protocol between included studies. Considering the particularity of acupuncture treatment, it is significant to synthesize the studies focusing on specific manipulation of acupuncture.

\section{Conclusion}

The meta-analyses of clinical outcome measures (FMA, NDS, BI, or MBI) show that acupuncture treatment is effective for motor function recovery in ischemic stroke patients. The ReHo meta-analysis reveals that acupuncture could induce extensive changes of cerebral activity, which suggests that the alterations of the basal ganglia, insula, and motorrelated areas are involved in neuroplasticity of acupuncture. These findings provide a new insight into the mechanisms underlying effectiveness of acupuncture and also lead to the articulation of a more general hypothesis that acupuncture plays a role in facilitating neuroplasticity.

\section{Conflicts of Interest}

The authors declare that there is no conflict of interest regarding the publication of this paper.

\section{Acknowledgments}

We thank Prof. Ying Zhang for the assistance. The study was supported by the National Natural Resources Fund (grant number 81873257) and Beijing Municipal Commission of Planning and Natural Resources (grant number 7182104).

\section{Supplementary Materials}

The supplementary material for this article can be found in the supplementary file. Figure S1A | Forest plot presenting the study meta-analysis for the living quality (nervous deficiency scale). Figure S1B | Forest plot presenting the study meta-analysis for the activity of daily living (Barthel index), Figure S1C | Forest plot presenting the study meta-analysis for the activity of daily living (modified Barthel index), Figure S1D | Forest plot presenting the study meta-analysis for the limb function (Fugl-Meyer assessment), Table S1A Metaregression analysis of FMA scores in treatment group, Table S1B Meta-regression analysis of age in treatment group. Table S1C Meta-regression analysis of duration in treatment group. (Supplementary Materials) 


\section{References}

[1] M. Zhou, H. Wang, X. Zeng et al., "Mortality, morbidity, and risk factors in China and its provinces, 1990-2017: a systematic analysis for the Global Burden of Disease Study 2017," The Lancet, vol. 394, no. 10204, pp. 1145-1158, 2019.

[2] J. SUN and W. CHEN, "Correlation studies of barium on pulmonary infection under the assessment of VFSS," Experimental and Therapeutic Medicine, vol. 11, no. 2, pp. 435438, 2016.

[3] S. Kumar, M. H. Selim, and L. R. Caplan, "Medical complications after stroke," The Lancet Neurology, vol. 9, no. 1, pp. 105-118, 2010.

[4] S. Li, Y.-T. Chen, G. E. Francisco, P. Zhou, and W. Z. Rymer, "A unifying pathophysiological account for post-stroke spasticity and disordered motor control," Frontiers in Neurology, vol. 10, p. 468, 2019.

[5] X. Tan, X. Liu, and H. Shao, "Healthy China 2030: a vision for health care," Value in Health Regional Issues, vol. 12, pp. 112114, 2017.

[6] J. Wang, J. Pei, D. Khiati et al., "Acupuncture treatment on the motor area of the scalp for motor dysfunction in patients with ischemic stroke: study protocol for a randomized controlled trial," Trials, vol. 18, no. 1, p. 287, 2017.

[7] M. Bonafede, A. Dick, K. Noyes, J. D. Klein, and T. Brown, "The effect of acupuncture utilization on healthcare utilization," Medical Care, vol. 46, no. 1, pp. 41-48, 2008.

[8] A. J. Vickers and K. Linde, "Acupuncture for chronic pain," Journal of the American Medical Association, vol. 311, no. 9, pp. 955-956, 2014.

[9] J. Wang, W. Shi, D. Khiati et al., Scalp acupuncture treatment for motor dysfunction in children with cerebral palsy: study protocol for a multicenter randomized controlled trial, vol. 21, no. 1, 2019, TRIALS, England, 2019.

[10] Z. Yang, J. Xie, and D. Liu, "Xingnao Kaiqiao needling method for acute ischemic stroke: a meta-analysis of safety and efficacy," Neural Regeneration Research, vol. 12, no. 8, pp. 13081314, 2017.

[11] X. He, Q. Sun, H. Liu, X. Guo, C. Chen, and L. Chen, “Timing of acupuncture during LTP-like plasticity induced by pairedassociative stimulation," Behavioural Neurology, vol. 2019, 10 pages, 2019.

[12] Y. Maeda, H. Kim, N. Kettner et al., "Rewiring the primary somatosensory cortex in carpal tunnel syndrome with acupuncture," Deutsche Zeitschrift für Akupunktur, vol. 60, no. 3, pp. 23-24, 2017.

[13] Y. Ning, K. Li, C. Fu et al., "Enhanced functional connectivity between the bilateral primary motor cortices after acupuncture at Yanglingquan (GB34) in right-hemispheric subcortical stroke patients: a resting-state fMRI study," Frontiers in Human Neuroscience, vol. 11, 2017.

[14] G. Jiang, X. Yin, C. Li et al., "The plasticity of brain gray matter and white matter following lower limb amputation," Neural Plasticity, vol. 2015, 10 pages, 2015.

[15] B. Dan, "Neuroscience underlying rehabilitation: what is neuroplasticity?," Developmental Medicine \& Child Neurology, vol. 61, no. 11, pp. 1240-1240, 2019.

[16] L. B. Reid, R. N. Boyd, R. Cunnington, and S. E. Rose, "Interpreting intervention induced neuroplasticity with fMRI: the case for multimodal imaging strategies," Neural Plasticity, vol. 2016, 13 pages, 2016.
[17] L. Cui, X. Gong, Y. Tang et al., "Relationship between the LHPP gene polymorphism and resting-state brain activity in major depressive disorder," Neural Plasticity, vol. 2016, 8 pages, 2016.

[18] B. Zhang, J. Lu, J. Xia et al., "Functional insights into aberrant brain responses and integration in patients with lifelong premature ejaculation,” Scientific Reports, vol. 7, no. 1, 2017.

[19] J. Zhao, D. Tomasi, C. E. Wiers et al., "Correlation between Traits of Emotion-Based Impulsivity and Intrinsic DefaultMode Network Activity," Neural Plasticity, vol. 2017, 9 pages, 2017.

[20] J. D. Schaechter, B. D. Connell, W. B. Stason et al., "Correlated change in upper limb function and motor cortex activation after verum and sham acupuncture in patients with chronic stroke," Journal of Alternative and Complementary Medicine, vol. 13, no. 5, pp. 527-532, 2007.

[21] P. N, "Application of ffMRI technique in evaluating electroacupuncture for ischemic stroke," Medical Innovation of China, vol. 16, no. 7, 2019.

[22] V. I. Müller, E. C. Cieslik, A. R. Laird et al., “Ten simple rules for neuroimaging meta-analysis," Neuroscience \& Biobehavioral Reviews, vol. 84, pp. 151-161, 2018.

[23] M. Tahmasian, A. A. Sepehry, F. Samea et al., "Practical recommendations to conduct a neuroimaging meta-analysis for neuropsychiatric disorders," Human Brain Mapping., vol. 40, no. 17, pp. 5142-5154, 2019.

[24] H. I. L. Jacobs, J. Radua, H. C. Lückmann, and A. T. Sack, "Meta-analysis of functional network alterations in Alzheimer's disease: toward a network biomarker," Neuroscience \& Biobehavioral Reviews, vol. 37, no. 5, pp. 753-765, 2013.

[25] B. Qin, M. X. Yang, W. Gao et al., "Voxel-wise meta-analysis of structural changes in gray matter of Parkinson's disease patients with mild cognitive impairment," Brazilian Journal of Medical and Biological Research, vol. 53, no. 6, p. e9275, 2020.

[26] S. Cheng, G. Xu, J. Zhou et al., "A multimodal meta-analysis of structural and functional changes in the brain of tinnitus," Frontiers in Human Neuroscience, vol. 14, p. 28, 2020.

[27] J. Li, P. Pan, R. Huang, and H. Shang, "A meta-analysis of voxel-based morphometry studies of white matter volume alterations in Alzheimer's disease," Neuroscience \& Biobehavioral Reviews, vol. 36, no. 2, pp. 757-763, 2012.

[28] J. Radua, D. Mataix-Cols, M. L. Phillips et al., "A new metaanalytic method for neuroimaging studies that combines reported peak coordinates and statistical parametric maps," European Psychiatry, vol. 27, no. 8, pp. 605-611, 2012.

[29] J. R. Dugré, J. Radua, M. Carignan-Allard, A. Dumais, K. Rubia, and S. Potvin, "Neurofunctional abnormalities in antisocial spectrum: a meta-analysis of fMRI studies on Five distinct neurocognitive research domains," Neuroscience \& Biobehavioral Reviews, vol. 119, pp. 168-183, 2020.

[30] A. Albajes-Eizagirre, A. Solanes, M. A. Fullana et al., "Metaanalysis of voxel-based neuroimaging studies using seed-based $\mathrm{d}$ mapping with permutation of subject images (SDM-PSI)," Journal of Visualized Experiments, no. 153, 2019.

[31] W. Ping, Z. Fang, L. Yongxin et al., "Effect of acupuncture plus conventional treatment on brain activity in ischemic stroke patients: a regional homogeneity analysis," Journal of Traditional Chinese Medicine, vol. 37, no. 5, pp. 650-658, 2017.

[32] C. Nana, Observation on the brain function in patients with hemiplegia treated by multiple acupuncture points, Guangzhou 
University of Traditional Chinese Medicine, Guangzhou, China, 2017.

[33] Y. Zhengfei and G. Rongai, "Effects of Yang elevating and Du meridian activating method in the treatment of stroke hemiplegia and the influence on resting - state functional brain imaging," Journal of Sichuan of Traditional Chinese Medicine, vol. 36, no. 8, pp. 186-188, 2018.

[34] Z. Yumei, Effects of acupuncture on brain activity in ischemic stroke patients- an regional homogeneity analysis, Chengdu University of Traditional Chinese Medicine, Sichuan, China, 2015.

[35] X. Ximei, W. Ping, H. Linna, and A. Junming, "FMRI research of acupuncture on brain regional homogeneity in resting-state of patients with ischemic stroke," Liaoning Journal of Traditional Chinese Medicine, vol. 40, no. 7, pp. 1287-1290, 2013.

[36] C. Zhihong, "A study of functional magnetic resonance imaging (fMRI) and the cinical effect in stroke patient using lifting yang to dredging du meridian manipulation," Yunnan University of Traditional Chinese Medicine, Yunnan, China, 2016.

[37] L. Lu, X.-g. Zhang, L. L. D. Zhong et al., “Acupuncture for neurogenesis in experimental ischemic stroke: a systematic review and meta-analysis," Scientific Reports, vol. 6, no. 1, 2016.

[38] L. Peng, C. Zhang, L. Zhou, H.-X. Zuo, X.-K. He, and Y.M. Niu, "Traditional manual acupuncture combined with rehabilitation therapy for shoulder hand syndrome after stroke within the Chinese healthcare system: a systematic review and meta-analysis," Clinical Rehabilitation, vol. 32 , no. 4, pp. 429439, 2018.

[39] Y. Dauvilliers and P. Peigneux, "Ictal SPECT in patients with rapid eye movement sleep behaviour disorder," Brain, vol. 138, no. 11, pp. e390-e390, 2015.

[40] V. K. Neychev, X. Fan, V. I. Mitev, E. J. Hess, and H. A. Jinnah, "The basal ganglia and cerebellum interact in the expression of dystonic movement," Brain, vol. 131, no. 9, pp. 2499-2509, 2008.

[41] W. You, O. Yanggang, Z. Xueling, Z. Minglan, and $X$. Shouyong, "Effects of acupuncture on brain regional homogeneity at resting state in the patients with ischemic stroke," Practical Geriatrics, vol. 29, no. 12, pp. 1010-1013, 2015.

[42] C.-. Q. Lu, W. Xu, C.-. H. Zeng et al., "Altered amplitude of low-frequency fluctuation in basal ganglia correlates to pulmonary ventilation function in COPD patients: a resting-state fMRI study," Brain and Behavior, vol. 9, no. 7, 2019.

[43] H. Man, Y. Bi, Y. Yu et al., "Associated factors of early neurological deterioration in isolated acute lacunar infarction in basal ganglia," Journal of Neurorestoratology, vol. 7, no. 2, pp. 63-69, 2019.

[44] A. Primaßin, N. Scholtes, S. Heim et al., "Determinants of concurrent motor and language recovery during intensive therapy in chronic stroke patients: four single-case studies," Frontiers in Neurology, vol. 6, 2015.

[45] Y.-C. Chen, C. Chen, R. M. Martínez, J. L. Etnier, and Y. Cheng, "Habitual physical activity mediates the acute exercise-induced modulation of anxiety-related amygdala functional connectivity," Scientific Reports, vol. 9, no. 1, p. 19787, 2019.

[46] R. Leigh, K. Oishi, J. Hsu et al., "Acute lesions that impair affective empathy," Brain, vol. 136, no. 8, pp. 2539-2549, 2013.

[47] R. P. Dum and P. L. Strick, "Motor areas in the frontal lobe of the primate," Physiology \& Behavior, vol. 77, no. 4-5, pp. 677682,2002 .
[48] A. S. Oliveira, B. R. Schlink, W. D. Hairston, P. König, and D. P. Ferris, "Restricted vision increases sensorimotor cortex involvement in human walking," Journal of Neurophysiology, vol. 118, no. 4, pp. 1943-1951, 2017.

[49] S. Tanaka, M. Honda, and N. Sadato, "Modality-specific cognitive function of medial and lateral human Brodmann area 6," The Journal of Neuroscience, vol. 25, no. 2, pp. 496-501, 2005.

[50] N. Dancause, "Extensive cortical rewiring after brain injury," The Journal of Neuroscience, vol. 25, no. 44, pp. 1016710179, 2005.

[51] C. M. Cirstea, W. M. Brooks, S. C. Craciunas et al., "Primary motor cortex in stroke," Stroke, vol. 42, no. 4, pp. 1004-1009, 2011.

[52] L. J. Volz, M. Vollmer, J. Michely, G. R. Fink, J. C. Rothwell, and C. Grefkes, "Time-dependent functional role of the contralesional motor cortex after stroke," NeuroImage: Clinical, vol. 16, pp. 165-174, 2017.

[53] M. F. Levin, J. A. Kleim, and S. L. Wolf, "What do motor "recovery" and "compensation" mean in patients following stroke?," Neurorehabilitation and Neural Repair, vol. 23, no. 4, pp. 313-319, 2009.

[54] E. MacDonald, H. Van der Lee, D. Pocock et al., "A novel phosphodiesterase type 4 inhibitor, HT-0712, enhances rehabilitation-dependent motor recovery and cortical reorganization after focal cortical ischemia," Neurorehabilitation and Neural Repair, vol. 21, no. 6, pp. 486-496, 2007.

[55] N. Dancause, S. Barbay, S. B. Frost et al., "Effects of small ischemic lesions in the primary motor cortex on neurophysiological organization in ventral premotor cortex," Journal of Neurophysiology, vol. 96, no. 6, pp. 3506-3511, 2006.

[56] S. H. Jang, "A review of the ipsilateral motor pathway as a recovery mechanism in patients with stroke," NeuroRehabilitation, vol. 24, no. 4, pp. 315-320, 2009.

[57] D. Tombari, I. Loubinoux, J. Pariente et al., "A longitudinal fMRI study: in recovering and then in clinically stable subcortical stroke patients," NeuroImage, vol. 23, no. 3, pp. 827839, 2004.

[58] L. M. Carey, D. F. Abbott, G. F. Egan, J. Bernhardt, and G. A. Donnan, "Motor impairment and recovery in the upper limb after stroke," Stroke, vol. 36, no. 3, pp. 625-629, 2005.

[59] A. K. Rehme, S. B. Eickhoff, C. Rottschy, G. R. Fink, and C. Grefkes, "Activation likelihood estimation meta-analysis of motor-related neural activity after stroke," NeuroImage, vol. 59, no. 3, pp. 2771-2782, 2012. 